\title{
SEMIPRIMARY HEREDITARY ALGEBRAS
}

\author{
BY
}

\section{ABRAHAM ZAKS}

\begin{abstract}
Let $\Sigma$ be a semiprimary $k$-algebra, with radical $M$. If $\Sigma$ admits a splitting then $\operatorname{dim}_{k} \Sigma / M \leqq \operatorname{dim}_{k} \Sigma$. The residue algebra $\Sigma / M^{2}$ is finite (cohomological) dimensional if and only if all residue algebras are finite dimensional. If $\operatorname{dim}_{k} \Sigma=1$ then all residue algebras are finite dimensional.
\end{abstract}

1. Introduction. We consider the following properties of algebras over a field $k$ :

(p1): $\operatorname{dim}_{k} \Sigma \leqq 1$.

(p2): $\operatorname{dim}_{k} \Sigma / I$ is finite for every two sided ideal $I$ in $\Sigma$.

(p3): $\operatorname{dim}_{k} \Sigma / M^{2}$ is finite, where $M$ is the (Jacobson) radical of $\Sigma$.

(p4): $\Sigma$ is a residue algebra of $\Omega$, where gl.dim $\Omega \leqq 1, \operatorname{dim}_{k} \Sigma / M$ is finite and $\Sigma / M$ is isomorphic to $\Omega / N, N$ being the (Jacobson) radical of $\Omega$.

(p5): $\Sigma$ is a residue algebra of $\Omega$, where $\operatorname{dim}_{k} \Omega \leqq 1$ and $\Sigma / M^{2}$ is isomorphic to $\Omega / N^{2}$.

For a finite dimensional $k$-algebra $\Sigma$, it was proved by Eilenberg, Nagao and Nakayama in [6] that $(\mathrm{p} 1) \Rightarrow(\mathrm{p} 2)$, while Jans and Nakayama proved in [7] the implications $(\mathrm{p} 3) \Rightarrow(\mathrm{p} 4) \Rightarrow(\mathrm{p} 5)$. Thus for finite dimensional $k$-algebras one has the equivalences $(\mathrm{p} 2) \Leftrightarrow(\mathrm{p} 3) \Leftrightarrow(\mathrm{p} 4) \Leftrightarrow(\mathrm{p} 5)$.

The purpose of this paper is to establish the implication $(\mathrm{p} 1) \Rightarrow(\mathrm{p} 2)$, and the equivalences $(\mathrm{p} 2) \Leftrightarrow(\mathrm{p} 3) \Leftrightarrow(\mathrm{p} 4)$ for semiprimary rings that are $k$-algebras. The equivalence $(\mathrm{p} 4) \Leftrightarrow(\mathrm{p} 5)$ can be deduced in certain particular cases as for instance if $\Sigma / M$ is a finite dimensional $k$-algebra. To this extent we give an example of a semiprimary ring $\Sigma$ for which $\operatorname{dim}_{k} \Sigma=1$ and $\operatorname{dim}_{k} \Sigma / M=1$.

As it turns out the passage from finite dimensional $k$-algebras to semiprimary ones is made possible by a lemma that seems to be of some interest in its own sake, namely:

A semiprimary $k$-algebra $\Sigma$ that admits a splitting $\Sigma=\Delta+M$ [9] satisfies the inequality $\operatorname{dim}_{k} \Delta \leqq \operatorname{dim}_{k} \Sigma$, where $\Delta$ denotes the residue algebra $\Sigma / M$.

In [2] Auslander proved that if $\Delta$ is a finite dimensional $k$-algebra and $\operatorname{dim}_{k} \Sigma$ is finite, then $\operatorname{dim}_{k} \Sigma=\operatorname{gl} \cdot \operatorname{dim} \Sigma$. He raised the problem whether it is necessary that $\operatorname{dim}_{k} \Delta=0$ (e.g. [4] and [5]). We prove the answer to be affirmative in case that $\operatorname{dim}_{k} \Sigma / M^{2}$ is finite.

Received by the editors February 27, 1970.

AMS 1969 subject classifications. Primary 1690; Secondary 1650.

Key words and phrases. Semiprimary hereditary algebra, splitting of a ring, finite dimensional algebra, separable algebra, ring of triangular matrices.

Copyright (C) 1971, American Mathematical Society 
2. Hereditary algebras. A $k$-algebra $\Sigma$ is said to be a semiprimary $k$-algebra if $\Sigma$ is a semiprimary ring, i.e. its (Jacobson) radical $M$ is nilpotent and the residue ring $\Sigma / M$ is a semisimple (Artinian) ring. Set $\Delta=\Sigma / M$. We say that a $k$-algebra $\Sigma$ is an hereditary $k$-algebra if $\operatorname{dim}_{k} \Sigma \leqq 1$. By $\Sigma^{\circ}$ we denote the apposite ring to $\Sigma$. By $(\Sigma: k)<\infty$ we denote that the $k$-algebra $\Sigma$ is finite dimensional (as a $k$-vector space). For the rest we write $\operatorname{dim}$ for $\operatorname{dim}_{k}$, and $\otimes$ for $\otimes_{k}$. We say that $\Sigma$ admits a splitting if $\Sigma=\Delta+M$ [7], [9]. A crucial step towards our main theorem is the following lemma:

LEMMA 1. If $\Sigma$ admits a splitting, $\Sigma=\Delta+M$, then $\operatorname{dim} \Delta \leqq \operatorname{dim} \Sigma$.

Proof. If $\operatorname{dim} \Sigma=\infty$ we are done. Otherwise $\operatorname{dim} \Sigma$ is finite, and we may assume that $\operatorname{dim} \Sigma=t<\infty$. By [4] we have the equality $\operatorname{gl} \cdot \operatorname{dim} \Sigma \otimes \Delta^{\circ}=\operatorname{dim} \Sigma=t<\infty$. Since $\Delta$ is a semisimple ring, $M$ is a projective right $\Delta$-module. From the natural isomorphism of $M \otimes \Delta^{\circ}$ with $M \otimes_{\Delta}\left(\Delta \otimes \Delta^{\circ}\right)$ it follows that $M \otimes \Delta^{\circ}$ is a projective right $\Delta \otimes \Delta^{\circ}$-module. Hence via the natural embedding of $\Delta \otimes \Delta^{\circ}$ into $\Sigma \otimes \Delta^{\circ}, \Sigma \otimes \Delta^{\circ}$ becomes a projective right $\Delta \otimes \Delta^{\circ}$-module. Denote by $f$ the natural embedding of $\Delta \otimes \Delta^{\circ}$ into $\Sigma \otimes \Delta^{\circ}$, and denote by $g$ the canonical epimorphism of $\Sigma \otimes \Delta^{\circ}$ onto $\Delta \otimes \Delta^{\circ}$, then $g \circ f$ is the identity map on $\Delta \otimes \Delta^{\circ}$.

For any left $\Delta \otimes \Delta^{\circ}$-module $A$ we set $A_{f}=\left(\Sigma \otimes \Delta^{\circ}\right) \otimes_{\left(\Delta \otimes \Delta^{\circ}\right)} A$.

For any left $\Sigma \otimes \Delta^{\circ}$-module $B$ we set $B_{g}=\left(\Delta \otimes \Delta^{\circ}\right) \otimes_{\left(\Sigma \otimes \Delta^{\circ}\right)} B$.

There results a $\Delta \otimes \Delta^{\circ}$ isomorphism from $A$ onto $\left(A_{f}\right)_{g}$, for every left $\Delta \otimes \Delta^{\circ}$ module $A$.

Let $A$ be a left $\Delta \otimes \Delta^{\circ}$-module, and let

$$
0 \rightarrow L \rightarrow P_{t-1} \rightarrow \cdots \rightarrow P_{0} \rightarrow A \rightarrow 0
$$

be an exact sequence of left $\Delta \otimes \Delta^{\circ}$-modules, where $P_{0}, \ldots, P_{t-1}$ are projective $\Delta \otimes \Delta^{\circ}$-modules. We claim that either $L$ is a projective $\Delta \otimes \Delta^{\circ}$-module, or else $L=0$.

Since $\Sigma \otimes \Delta^{\circ}$ is a projective right $\Delta \otimes \Delta^{\circ}$-module, there results an exact sequence of left $\Sigma \otimes \Delta^{\circ}$-modules:

$$
0 \rightarrow L_{f} \rightarrow\left(P_{t-1}\right)_{f} \rightarrow \cdots \rightarrow\left(P_{0}\right)_{f} \rightarrow A_{f} \rightarrow 0
$$

where $\left(P_{i}\right)_{f}=\left(\Sigma \otimes \Delta^{\circ}\right) \otimes_{\left(\Delta \otimes \Delta^{\circ}\right)} P_{i}$, for $i=0, \ldots,(t-1)$. Thus $\left(P_{0}\right)_{f, \ldots,}\left(P_{t-1}\right)_{f}$ are $\Sigma \otimes \Delta^{\circ}$-projective. Since 1.gl.dim $\Sigma \otimes \Delta^{\circ}=\operatorname{dim} \Sigma=t$, it follows that either $L_{f}$ is a projective $\Sigma \otimes \Delta^{\circ}$-module, or else $L_{f}=0$. Hence $L$ is $\Delta \otimes \Delta^{\circ}$ isomorphic to the $\Delta \otimes \Delta^{\circ}$-module $\left(L_{f}\right)_{g}=\left(\Delta \otimes \Delta^{\circ}\right) \otimes_{\left(\Sigma \otimes \Delta^{\circ}\right)} L_{f}$. Therefore $L=0$ or else $L$ is a projective $\Delta \otimes \Delta^{\circ}$-module.

Therefore for every left $\Delta \otimes \Delta^{\circ}$-module $A$ we have 1.p.dim $\operatorname{dim}_{\Delta \Delta^{\circ}} \mathrm{A} \leqq t$, thus 1.gl.dim $\Delta \otimes \Delta^{\circ} \leqq t$. Since by [4] we have the equality $\operatorname{dim} \Delta=1 . g l . \operatorname{dim} \Delta \otimes \Delta^{\circ}$ we may conclude that the inequality $\operatorname{dim} \Delta \leqq \operatorname{dim} \Sigma$ holds.

Recall that all residue rings of a semiprimary ring $\Sigma$ are of finite global dimension iff $\Sigma$ is a residue ring of a semiprimary ring $\Omega$ for which gl.dim $\Omega \leqq 1$, and this 
is the case iff gl.dim $\Sigma / M^{2}$ is finite [9]. Under each of these equivalent conditions $\Sigma$ admits a splitting $\Sigma=\Delta+M$.

The splitting of $\Sigma$ is inherited by every residue ring $\Sigma_{1}$ of $\Sigma, \Sigma_{1}=\Delta_{1}+M_{1}$. Furthermore, $\Delta_{1}$ is (up to an isomorphism) a direct factor of $\Delta$.

We are now ready to state and prove our main theorem that establishes the equivalences $(\mathrm{p} 2) \Leftrightarrow(\mathrm{p} 3) \Leftrightarrow(\mathrm{p} 4)$ for semiprimary $k$-algebras.

THEOREM 1. The following are equivalent:

(a) $\operatorname{dim} \Delta<\infty$ and gl.dim $\Sigma / M^{2}<\infty$.

(b) $\operatorname{dim} \Sigma / I<\infty$ for every two sided ideal I in $\Sigma$.

(c) $\operatorname{dim} \Sigma / M^{2}<\infty$.

Proof. (a) $\Rightarrow$ (b): From gl.dim $\Sigma / M^{2}<\infty$ it follows by [9] that gl.dim $\Sigma / I<\infty$ for every two sided ideal $I$ in $\Sigma$. Set $\Delta_{1}=(\Sigma / I) /(I+M / I)$ then $\Delta_{1}$ is a direct factor of $\Delta$, hence $\operatorname{dim} \Delta_{1} \leqq \operatorname{dim} \Delta$. Combining the equality $\operatorname{dim} \Sigma / I=1$. gl.dim $\Sigma / I \otimes \Delta_{1}^{\circ}$ [4] with the inequality l.gl.dim $\Sigma / I \otimes \Delta_{1}^{\circ} \leqq 1 . g l \cdot \operatorname{dim} \Sigma / I+\operatorname{dim} \Delta_{1}^{\circ}$ [5] it results that $\operatorname{dim} \Sigma / I$ is finite.

(b) $\Rightarrow$ (c) is obvious.

(c) $\Rightarrow$ (a): Since gl.dim $\Sigma / M^{2} \leqq \operatorname{dim} \Sigma / M^{2}$ [5], then $\operatorname{gl} \cdot \operatorname{dim} \Sigma / M^{2}<\infty$. Hence by [9] $\Sigma / M^{2}$ admits a splitting, and thus Lemma 1 implies the inequality $\operatorname{dim} \Delta \leqq$ $\operatorname{dim} \Sigma / M^{2}$, therefore $\operatorname{dim} \Delta$ is finite.

Observe that under each of the equivalent conditions in Theorem $1, \Sigma$ is a residue of a semiprimary $k$-algebra $\Omega$ with radical $N$, such that $\Omega / N$ is isomorphic with $\Delta$, and gl.dim $\Omega \leqq 1$. This is an immediate consequence of Theorem 1 applied to $\Omega(\Delta, N)$ [9]. It is worth noticing that $\Sigma$ admits a splitting, $\Sigma=\Delta+M$.

As for $\operatorname{dim} \Omega$, from $\operatorname{dim} \Omega=1 . g 1 . \operatorname{dim} \Omega \otimes \Delta^{\circ}$ it follows that $\operatorname{dim} \Delta \leqq \operatorname{dim} \Omega \leqq$ $\operatorname{dim} \Delta+1$.

In the next section we will bring some examples showing that it is possible that $\operatorname{dim} \Omega=\operatorname{dim} \Delta+1$, but it is also possible that the equality $\operatorname{dim} \Omega=\operatorname{dim} \Delta$ will hold.

Consider the case where $k$ is the center of $\Sigma$. One can easily construct examples in which $\Sigma$ is a residue ring of a semiprimary hereditary ring $\Omega$ with radical $N$, such that $\Omega / N$ is isomorphic with $\Delta$, but $\Omega$ is not a $k$-algebra, i.e., not every semiprimary hereditary ring-of which $\Sigma$ is a residue ring-is a $k$-algebra [10, Example 1].

Notice that if $\Delta$ is a finite dimensional $k$-algebra then $\operatorname{dim} \Delta=0$. One verifies that if $M \neq 0$ then $\operatorname{dim} \Omega=1$. Furthermore, if $\Omega$ is any semiprimary hereditary ring with radical $N$ of which $\Sigma$ is a residue ring, such that $\Omega / N$ is isomorphic with $\Delta$, then $\Omega$ admits a splitting, $\Omega=\Delta+A+N^{2}$. Therefore, if one insists on $\Omega / N^{2}$ being isomorphic to $\Sigma / M^{2}$ it follows that up to an isomorphism $\Omega$ is uniquely determined. This establishes the equivalence $(\mathrm{p} 4) \Leftrightarrow(\mathrm{p} 5)$ in case $\Delta$ is a finite dimensional $k$-algebra. Also in this case we have $\operatorname{dim} \Omega / I=\operatorname{gl} \operatorname{dim} \Omega / I$ for every two sided ideal $I$ in $\Omega$. In particular from [10] it results that $\operatorname{dim} \Omega / I \leqq \operatorname{dim} \Omega / N^{2}$, whenever $I \subset N^{2}$. 
We do not know if this last inequality holds without the assumption $\operatorname{dim} \Delta=0$. Our next aim is to prove the implication $(\mathrm{p} 1) \Rightarrow(\mathrm{p} 2)$ for semiprimary $k$-algebras. Recall that the validity of this implication for finite dimensional $k$-algebras is based on the equality $\operatorname{dim} \Sigma=\operatorname{gl} \operatorname{dim} \Sigma$, which is a consequence of $\operatorname{dim} \Delta=0$ under these circumstances (e.g. [6]). For semiprimary $k$-algebras we have by [5] the inequality gl.dim $\Sigma \leqq \operatorname{dim} \Sigma$. Furthermore, if gl.dim $\Sigma / M^{2}$ is finite then by [9] $\Sigma$ admits a splitting $\Sigma=\Delta+M$. We proceed with a sequence of corollaries to get the desired implication.

COROLlaRY 1. If $\Sigma$ admits a splitting, $\Sigma=\Delta+M$, then $\operatorname{dim} \Delta \leqq \operatorname{dim} \Sigma / I$ for every two sided ideal I in $\Sigma$ that is contained in the radical.

Proof. Since the splitting is inherited by all residue rings of $\Sigma$, and since $I \subset M$ implies that $(\Sigma / I) /(I+M / I)$ is isomorphic with $\Delta$, then we have applying Lemma 1: $\operatorname{dim} \Delta \leqq \operatorname{dim} \Sigma / I$.

COROllary 2. If $M^{2}=0$ then $\operatorname{dim} \Delta \leqq \operatorname{dim} \Sigma$.

Proof. If $\operatorname{dim} \Sigma=\infty$ we are done. Otherwise $\operatorname{dim} \Sigma$ is finite, hence $\operatorname{gl}$.dim $\Sigma$ is finite. Therefore $\Sigma$ admits a splitting, $\Sigma=\Delta+M$, and the result follows from Lemma 1.

Corollary 3. If $\operatorname{dim} \Sigma=1$ then $\operatorname{dim} \Delta \leqq 1$.

Proof. The proof is an immediate consequence of Lemma 1 which is applicable in this case, since gl.dim $\Sigma \leqq \operatorname{dim} \Sigma=1$ implies the splitting of $\Sigma$.

It seems interesting to notice that one can prove that $\operatorname{dim} \Delta$ is finite by observing that $\Delta \otimes \Delta^{\circ}$ is a residue ring of the hereditary ring $\Sigma \otimes \Delta^{\circ}$ by the nilpotent two sided ideal $M \otimes \Delta^{\circ}$ (e.g. [6]).

As a consequence there results the implication $(\mathrm{p} 1) \Rightarrow(\mathrm{p} 2)$.

Corollary 4. If $\operatorname{dim} \Sigma=1$ then $\operatorname{dim} \Sigma / I$ is finite for every two sided ideal I in $\Sigma$.

Proof. This is an immediate consequence of Theorem 1 since by Corollary 3 $\operatorname{dim} \Delta \leqq 1$, and since $\operatorname{gl} \operatorname{dim} \Sigma \leqq \operatorname{dim} \Sigma$ implies that $\operatorname{gl} . \operatorname{dim} \Sigma / M^{2}$ is finite.

3. Examples. In this section we will bring some examples of $k$-algebras, all the residue algebras of which have finite cohomological dimension. We will be mainly concerned with the inequalities $\operatorname{dim} \Delta \leqq \operatorname{dim} \Sigma \leqq \operatorname{dim} \Delta+\operatorname{gl} \operatorname{dim} \Sigma$, and with the equality $\operatorname{dim} \Sigma=$ gl.dim $\Sigma$ without $(\Delta: k)$ being finite.

Let $k\left(x_{1}, \ldots, x_{n}, y_{1}, \ldots, y_{m}\right)$ be the field of rational functions in $n+m$ variables over the field $k$. We will identify $k\left(k\left(x_{1}, \ldots, x_{n}\right), k\left(y_{1}, \ldots, y_{m}\right)\right)$ with its natural embedding in $k\left(x_{1}, \ldots, x_{n}, y_{1}, \ldots, y_{m}\right)$.

EXAMPLE 1. Let $\Sigma$ be the $k$-subalgebra of the $2 \times 2$ matrix algebra over the field of rational functions in one variable over a field $k, k(x)$. A matrix $\sigma$ belongs to $\Sigma$ iff $\sigma$ is of the form

$$
\left\|\begin{array}{ll}
a & 0 \\
b & c
\end{array}\right\|
$$

where $a$ is an element of $k$, and $b, c$ are elements of $k(x)$. 
Obviously $\Sigma$ is a left Artinian hereditary ring with radical $M$ of square zero, and $\operatorname{dim} \Delta=1$. We claim that $\operatorname{dim} \Sigma=1$. It will suffice to show that $\operatorname{lgl} \operatorname{ld} \operatorname{dim} \Sigma \otimes k(x)=$ 1. Identify $\Sigma \otimes k(x)$ with a subring of the $2 \times 2$ matrix algebra over $k(x) \otimes k(x)$, namely: $\sigma^{\prime}$ belongs to $\Sigma \otimes k(x)$ iff $\sigma^{\prime}$ is of the form:

$$
\left\|\begin{array}{ll}
\alpha & 0 \\
\beta & \gamma
\end{array}\right\|,
$$

where $\alpha$ belongs to $k \otimes k(x)$ (which is isomorphic to $k(x)$ ), and $\beta, \gamma$ belong to $k(x) \otimes k(x)$.

Let $J$ be a left ideal in $\Sigma \otimes k(x)$, then one readily verifies that $J$ is of one of the following two types:

Type 1. Every element in $J$ is of the form

$$
\left\|\begin{array}{ll}
0 & 0 \\
\beta & \gamma
\end{array}\right\| \text {. }
$$

Type 2. $J$ is a direct sum of two subideals $J_{1}$ and $J_{2}$ where every element of $J_{1}$ is of the form

$$
\left\|\begin{array}{ll}
0 & 0 \\
0 & \gamma
\end{array}\right\|
$$

and a matrix $\sigma^{\prime}$ belongs to $J_{2}$ iff it is of the form

$$
\left\|\begin{array}{ll}
\alpha & 0 \\
\beta & 0
\end{array}\right\| .
$$

Since $\operatorname{dim} \mathrm{k}(x)=1$ it follows from [3, Theorem 5.4, p. 14] that in either case $J$ is a projective left $\Sigma \otimes k(x)$-module. Hence by [3, Theorem 5.4, p. 14] it follows that $\operatorname{l.gl} \cdot \operatorname{dim} \Sigma \otimes k(x)=1$.

A similar treatment, using the fact that $k\left(x_{1}, \ldots, x_{n}\right) \otimes k\left(x_{1}, \ldots, x_{n}\right)$ is a Neotherian ring-where $k\left(x_{1}, \ldots, x_{n}\right)$ is the field of rational functions in $n$ variables over $k$-gives:

EXAMPLE $1^{*}$. Let $\Sigma$ be the $k$-subalgebra of the $2 \times 2$ matrix algebra over $k\left(x_{1}, \ldots, x_{n}\right)$. A matrix $\sigma$ belongs to $\Sigma$ iff $\sigma$ is of the form

$$
\left\|\begin{array}{ll}
a & 0 \\
b & c
\end{array}\right\|,
$$

where $a$ is an element of $k$, and $b, c$ are elements of $k\left(x_{1}, \ldots, x_{n}\right)$.

$\Sigma$ is a left Artinian ring with radical $M$ of square zero, $\operatorname{dim} \Delta=n$ [5], and $\operatorname{gl} . \operatorname{dim} \Sigma$ $=1$. Finally, by the remark made above, we have by checking $\operatorname{dim} \Sigma$ via $1 . g l \cdot d i m \Sigma$ $\otimes k\left(x_{1}, \ldots, x_{n}\right)$ that $\operatorname{dim} \Sigma=n$.

EXAMPLE 2. By taking successive rings of triangular matrices of the ring $\Sigma$ that was constructed in Example $1\left(1^{*}\right)$ we obtain a left Artinian ring $\Lambda=T_{n_{t}}\left(\cdots T_{n_{1}}(\Sigma) \cdots\right)$. By [5] it follows that

$$
\operatorname{dim} \Lambda=\text { gl.dim } \Lambda=t+1 \quad(\operatorname{dim} \Lambda=t+n, \operatorname{gl} \cdot \operatorname{dim} \Lambda=t+1) .
$$


Furthermore, if $N$ is the radical of $\Lambda$ then $\operatorname{dim} \Lambda / N=\operatorname{dim} \Delta$, since $\Lambda / N$ is isomorphic to a direct product of $n_{1} \cdots n_{t}$ copies of $\Delta$.

Summarizing we have:

Proposition 1. For every pair of positive integers $n, s$ there exists a $k$-algebra $\Sigma$ for which gl.dim $\Sigma=s, \operatorname{dim} \Delta=n$, and $\operatorname{dim} \Sigma<\operatorname{gl} \cdot \operatorname{dim} \Sigma+\operatorname{dim} \Delta$.

Taking $n=1$ there will result a $k$-algebra $\Sigma$ for which $\operatorname{dim} \Sigma=\operatorname{gl} . \operatorname{dim} \Sigma<\infty$, such that $\operatorname{dim} \Delta=1$.

EXAMPLE 3. Let $\Sigma$ be the $k$-subalgebra of the $2 \times 2$ matrix algebra over $k(x, y)$ the field of rational functions in two variables over the field $k$. A matrix $\sigma$ belongs to $\Sigma$ iff $\sigma$ is of the form

$$
\left\|\begin{array}{ll}
a & 0 \\
b & c
\end{array}\right\|,
$$

where $a$ belongs to $k(y), b$ belongs to $k(x, y)$, and $c$ belongs to $k(x)$.

$\Sigma$ is a semiprimary ring with radical of square zero. Obviously $\operatorname{dim} \Delta=1$, $\operatorname{gl} \cdot \operatorname{dim} \Sigma=1$, and it is an easy exercise to check that $\operatorname{dim} \Sigma=2$.

EXAMPLE $3 *$. Take $\Sigma$ to be the $k$-subalgebra of the $2 \times 2$ matrix algebra over $k\left(x_{1}, \ldots, x_{n}, y_{1}, \ldots, y_{n}\right)$-the field of rational functions in $2 n$ variables over the field $k$. A matrix $\sigma$ belongs to $\Sigma$ iff $\sigma$ is of the form

$$
\left\|\begin{array}{ll}
a & 0 \\
b & c
\end{array}\right\|
$$

where $a$ belongs to $k\left(y_{1}, \ldots, y_{n}\right), b$ belongs to $k\left(x_{1}, \ldots, x_{n}, y_{1}, \ldots, y_{n}\right)$, and $c$ belongs to $k\left(x_{1}, \ldots, x_{n}\right)$.

$\Sigma$ is a semiprimary ring, and gl.dim $\Sigma=1$. Again by straightforward computations it follows that $\operatorname{dim} \Sigma=n+1$, and from [5] $\operatorname{dim} \Delta=n$.

EXAMPLE 4. By taking successive rings of triangular matrices of the ring $\Sigma$ that was constructed in Example $3\left(3^{*}\right)$ we obtain a semiprimary ring $\Lambda=$ $T_{n_{t}}\left(\cdots T_{n_{1}}(\Sigma) \cdots\right)$ with radical $N$. From [5] it follows that gl.dim $\Lambda=t+1$, and $\operatorname{dim} \Lambda=t+2(\operatorname{dim} \Lambda=t+n+1)$. Furthermore, since $\Lambda / N$ is the direct product of $n_{1} \cdots n_{t}$ copies of $\Delta, \operatorname{dim} \Lambda / N=1(\operatorname{dim} \Lambda / N=n)$.

Summarizing we obtain:

Proposition 2. For every pair of positive integers $n, s$ there exists a $k$-algebra $\Sigma$ for which gl.dim $\Sigma=s, \operatorname{dim} \Delta=n$, and $\operatorname{dim} \Sigma=\operatorname{gl} \cdot \operatorname{dim} \Sigma+\operatorname{dim} \Delta$.

Notice that in all our examples, $k$ is the center of each of the constructed rings.

4. Applications. In [2] Auslander proved that if $\operatorname{dim} \Sigma<\infty$ and if $(\Delta: k)<\infty$ then $\operatorname{dim} \Sigma=\operatorname{gl} \operatorname{dim} \Sigma$. He raised the problem whether $\operatorname{dim} \Sigma$ is necessarily zero. In [4] Eilenberg proved that if $\operatorname{dim} \Sigma<\infty$ and $(\Sigma: k)<\infty$ then $\operatorname{dim} \Delta=0$ and $\operatorname{dim} \Sigma=\operatorname{gl} \cdot \operatorname{dim} \Sigma$. In $\S 3$ we saw that it is possible to have $\operatorname{dim} \Sigma<\infty$ and $\operatorname{dim} \Sigma=$ gl. $\operatorname{dim} \Sigma$ without $(\Sigma: k)$ nor $(\Delta: k)$ being finite. Furthermore, $\operatorname{dim} \Sigma=\operatorname{gl} . \operatorname{dim} \Sigma$ may hold without $\operatorname{dim} \Delta$ being zero. Still we have: 
Proposition 3. If $M^{2}=0$, if $(\Delta: k)<\infty$, and if $\operatorname{dim} \Sigma$ is finite then $\operatorname{dim} \Delta=0$.

Proof. By Corollary $2 \operatorname{dim} \Delta \leqq \operatorname{dim} \Sigma<\infty$. Since $(\Delta: k)<\infty$ we now have $\operatorname{dim} \Delta=0$.

In this respect it is worth stating an immediate consequence of Theorem 1, that turns out to be just an affirmative answer to the problem raised by Auslander in a particular case.

COROLlaRY 5. If $(\Delta: k)<\infty$ then the following are equivalent:

(a) $\operatorname{dim} \Delta=0$ and gl.dim $\Sigma / M^{2}<\infty$,

(b) $\operatorname{dim} \Sigma / M^{2}<\infty$.

Under each of these equivalent conditions $\operatorname{dim} \Sigma / I=\operatorname{gl} \operatorname{dim} \Sigma / I<\infty$ for every two sided ideal I in $\Sigma$.

Let $\Sigma_{1}\left(\Sigma_{2}\right)$ be a semiprimary $k$-algebra with radical $M_{1}\left(M_{2}\right)$, and set $\Delta_{i}=\Sigma_{i} / M_{i}$ for $i=1,2$. Assuming that $\left(\Delta_{i}: k\right)<\infty$, and $\operatorname{dim} \Sigma_{i} / M_{i}^{2}<\infty$ for $i=1,2$ it follows that $\operatorname{dim} \Delta_{i}=0$ for $i=1,2$. Denote $\Delta=\Delta_{1} \otimes \Delta_{2}$, and $N=M_{1} \otimes \Delta_{2}+\Delta_{1} \otimes M_{2}$ then it readily follows that $\operatorname{dim} \Omega \leqq 1$, where $\Omega=\Omega(\Delta, N)$ [9]. Furthermore, $\Sigma_{1} \otimes$ $\Sigma_{2}$ is a residue $k$-algebra of $\Omega$, and $(\Delta: k)<\infty$. We therefore have:

THEOREM 2. The class of semiprimary $k$-algebras $\mathfrak{E}$ is closed under tensor products. A semiprimary $k$-algebra $\Sigma$ belongs to $\mathbb{E}$ iff $\operatorname{dim} \Sigma / M^{2}<\infty$ and $(\Delta: k)<\infty$.

Notice that this theorem is no longer valid if we replace $\operatorname{dim} \Sigma / M^{2}<\infty$ by gl.dim $\Sigma / M^{2}<\infty$.

\section{REFERENCES}

1. M. Auslander, On the dimension of modules and algebras. III: Global dimension, Nagoya Math. J. 9 (1955), 67-77. MR 17, 579.

2. - On the dimension of modules and algebras. VI: Comparison of global and algebra dimension, Nagoya Math. J. 11 (1957), 61-65. MR 19, 14.

3. H. Cartan and S. Eilenberg, Homological algebra, Princeton Univ. Press, Princeton, N. J., 1956. MR 17, 1040.

4. S. Eilenberg, Algebras of cohomologically finite dimension, Comment. Math. Helv. 28 (1954), 310-319. MR 16, 442.

5. S. Eilenberg, A. Rosenberg and D. Zelinsky, On the dimension of modules and algebras. VIII: Dimension of tensor products, Nagoya Math. J. 12 (1957), 71-93. MR 20 \#5229.

6. S. Eilenberg, H. Nagao and T. Nakayama, On the dimension of modules and algebras. IV: Dimension of residue rings of hereditary rings, Nagoya Math. J. 10 (1956), 87-95. MR 18, 9.

7. J. P. Jans and T. Nakayama, On the dimension of modules and algebras. VII: Algebras with finite-dimensional residue-algebras, Nagoya Math. J. 11 (1957), 67-76. MR 19, 250.

8. I. Kaplansky, Modules over Dedekind rings and valuation rings, Trans. Amer. Math. Soc. 72 (1952), 327-340. MR 13, 719.

9. A. Zaks, Residue rings of semi-primary hereditary rings, Nagoya Math. J. 30 (1967), 279-283. MR 37 \#1409.

10. — Semi-primary hereditary rings, Israel J. Math. 6 (1968), 359-362.

Technion, IsRael Institute of Technology, Haifa, ISRAEL 